\title{
EGEC: An Energy Efficient Exponentially Generated Clustering Mechanism for Reactive Wireless Sensor Networks
}

\author{
Sundareswaran $\mathrm{P}^{\mathrm{a}}$, Rajesh $\mathrm{R} \mathrm{S}^{\mathrm{b}}$, Vardharajulu $\mathrm{K} \mathrm{N}^{\mathrm{c}}$ \\ ${ }^{a, b, c}$ Department of Computer Science and Engineering, Manonmaniam Sundaranar \\ University, Tirunelveli,627012, India.
}

Received: 17 March 2017; Accepted: 06 August 2018; Published: 08 November 2018

\begin{abstract}
As most of the wireless sensor networks are unattended by humans, the energy is an important issue while deploying nodes of wireless sensor network in hazardous environments. One of the method to preserve energy consumption among sensor nodes is clustering. Using clustering, the nodes send the data to the nearest cluster head instead of far away sink. In applications using reactive wireless sensor networks, a limited number of sensor nodes are activated due to exceed in threshold values and the other nodes are under idle state. As far as the reactive wireless sensor network is concerned, the number of clusters would have been based on the number of active nodes and alive nodes. In this work, an optimum number of clusters is computed based on the ratio between active nodes and alive nodes. Extensive analysis and comparisons are made with existing protocol and found that the proposed methodology outperforms the existing protocol in terms of networks life time and throughput.
\end{abstract}

Index Terms: Wireless Sensor Network; EGEC;TEEN, Routing; Clustering Protocol

(C) 2018 Published by MECS Publisher. Selection and/or peer review under responsibility of the Research Association of Modern Education and Computer Science

\section{Introduction}

In modern human life, technology and innovations are playing major role in smart living. Wireless sensors networks (WSNs) are now become integral part of human life. Most of the applications like transportation, smart grids, weather forecasting, environment monitoring, battle field surveillance, health monitoring and IoT are having the role of wireless Sensor Networks (WSNs).[1-8].

Being low cost and having sensing capability, sensors are widely used in the day to day life of human. A large number of tiny, energy constrained sensors are connected together to form a wireless sensor network. The sensors sense analog information from the environment where they are deployed and convert it into digital 
data before sending to the sink or base station.

The constraints such as coverage, energy, localization, data security play major role in designing the wireless sensor networks in a better way. The key issue among them is energy, since the nodes are provided with limited battery power and mostly unattended. The sensor nodes are deployed in hazardous environments like nuclear reactors and human unattended areas like forest. Therefore energy is an important criterion while designing a WSN. The common ways of consuming energy by a sensor node are during data reception, transmission and data aggregation. These ways of energy consumption leads to the death of the sensor nodes. Therefore the network life is also affected. In order to prolong the network life time, a lot of energy conservation techniques are applied in WSNs [12]. Data aggregation technique is used to avoid sending of the redundant data [9-10].

When all the sensor nodes send their data to the sink, the network traffic is increased tremendously. The far away nodes need multi hoping to send the data to the sink. Therefore the nodes near to the sink will become dead due to excessive forwarding of data to the base station. To avoid this, clustering technique has been introduced in WSNs. The entire sensor network has been divided into number of disjoint sets called clusters and each cluster has its own cluster head. The nodes belong to the respective cluster send data to its cluster head instead of sending directly to the sink. The network overhead in therefore considerably reduced and energy of each node is preserved.

Researchers are motivated to develop clustering algorithms and a lot of clustering algorithms have been developed for WSNs [14-16, 18-21]. Based on the functionalities, WSNs are grouped into two categories namely homogeneous and heterogeneous WSNs. The homogeneous WSNs have nodes with identical initial energy level. On the other hand the heterogeneous WNSs have sensor nodes with different energy levels and other dissimilar parameters. Popular protocols like LEACH [18], TEEN [20], PEGASIS [19] are examples of clustering protocols used in homogeneous WSNs. SEP [16], DEEC [23] are used in heterogeneous WSNs. The common feature available in the clustering protocols is data aggregation and using that the redundant data have been eliminated from transmission [11, 17].

The clustering algorithms have two phases namely set up phase and steady state phase. The clusters are formed and cluster heads are elected in set up phase and the member nodes are joined to the clusters in this phase. The sensed data are transmitted in steady state phase according to TDMA schedules sent by the cluster head.

The WSNs are classified into two categories as reactive and proactive networks based on functioning mode and the type of application. The nodes in the proactive WSN periodically sense the environment and transmit the data to the sink. Therefore the sensors and transmitters are always switched on and send the sensed parameter values at regular intervals. This kind of WSNs is mostly suitable for periodic monitoring applications. In the reactive networks, the nodes react immediately to drastic variation in the value of the sensed data. Therefore this kind of networks is mostly fitted for time critical applications. Almost all the clustering protocols compute the number of clusters based on the probability ratio, so that the cluster count is constant. In this proposed model, an exponential function is used to compute the optimum number of clusters. It is derived from the number of active nodes and the total number of alive nodes in the network at a particular instant. By using the optimum number of clusters at each round, the energy consumption is reduced considerably and the lifetime of the network is increased. The remaining part of the paper is organized as follows. Related works are discussed in section 2, section 3 describes the proposed methodology, simulation results are discussed in section 4 and section 5 concludes the paper.

\section{Related Works}

Since energy is the important issue in the design of a WSN, the researchers give much attention to energy saving mechanisms while developing the communication protocols for the WSN. One of the important protocols used in Wireless Sensor Networks (WSNs) is LEACH [18]. The LEACH Protocol efficiently manages energy conservation among the nodes by using randomized rotation of cluster heads among the nodes. 
This protocol also supports data aggregation and fusion technique. This technique combines the multiple redundant data into a single data and this data is sent to the base station by the cluster heads, so that multiple transmissions of similar data are reduced. In the LEACH protocol, the sensor nodes elect cluster heads (CHs) among $n$ nodes at regular time interval using the given probability. The probability threshold function for a node being as a cluster head is defined as-

$$
\mathrm{T}_{(n)}=\left\{\begin{array}{lr}
\frac{\mathrm{p}}{1-\mathrm{p} *\left(\operatorname{r\operatorname {mod}} \frac{1}{\mathrm{p}}\right)} & \text { if } \mathrm{n} \in \mathrm{G} \\
0 & \text { Otherwise }
\end{array}\right.
$$

Where $\mathrm{P}$ is the percentage of cluster heads among all nodes, $\mathrm{n}$ is the number of nodes present in the sensor network, and $\mathrm{r}$ is the number of rounds for the election. The value ( $\mathrm{r} \bmod 1 / \mathrm{P})$ is the number of nodes elected as cluster head in a cycle, and $\mathrm{G}$ is the set of nodes not elected as a cluster head in previous rounds. Nodes will be elected as cluster heads, when its randomly generated value is less than probability threshold value $T(n)$. In $\mathrm{LEACH}$, the number of cluster is computed based on the probability ratio in each round.

Threshold Sensitive Energy Efficient Sensor Network (TEEN) [20] is one of the protocols used for reactive WSNs. At every change of cluster cycle in TEEN, apart from the attribute to be sensed, sensor nodes know two threshold values.

Hard Threshold: If the sensed value is beyond the hard threshold value, the node transmits the data immediately to its cluster head.

Soft Threshold: This is the maximum variation between the sensed value and current value, beyond which the node triggers to switch on its transmitter.

In a real time application having sensor network with thousands of nodes, only the limited number of nodes are get activated simultaneously due to sudden change in the environment. Remaining number of nodes may be idle state and this is the strength of the TEEN protocol. The TEEN protocol also uses the probability ratio to calculate the number of cluster in each round.

A three way message exchange method is used in [15] among the sensor node with its neighbors to elect the cluster head. A node will be elected as cluster head based on its residual energy and its degree.

The intra-cluster communication cost is considered for selecting the cluster head in each round in [14]. In HEED a heterogeneous WSN protocol proposed by Younis and Fahmy, along with intra-cluster communication cost the node's residual energy is calculated for each round before electing the cluster head. The probability to become a cluster head for a node is computed as

$$
\mathrm{CH}_{\text {Prob }}=\mathrm{C}_{\text {Prob }} \frac{E_{\text {residual }}}{E_{\max }}
$$

where $\mathrm{C}_{\text {Prob }}$ is the optimal percentage of $\mathrm{CH}$ (Cluster Head) among all nodes in a network, $E_{\text {residual }}$ is the energy of the node at the corresponding round and Emax is a reference maximum energy which is equal for all the nodes in the network. $\mathrm{CH}_{\text {Prob }}$ will not be allowed below a threshold value. The two types of status send by a node to its neighbors are tentative status and final status. If the $\mathrm{CH}_{\text {Prob }}$ value of a node is less than 1, then the node becomes a tentative $\mathrm{CH}$ and this node will be converted into a normal node at a later iteration if it finds a low cost $\mathrm{CH}$. If the $\mathrm{CH}_{\text {Prob }}$ has reached 1 , the corresponding node permanently becomes a $\mathrm{CH}$. A chain based path construction is made in PEGASIS [19] from the farthest node to the closest node of a sink using the greedy algorithm. The data sensed by the far away node sends the data to the neighbor, which fuses the received data with its own one and send to the neighbor. This process is repeated until the data reached the sink. The leader node is considered as cluster head here.

The initial energy and residual energy levels of the sensor nodes are considered in the election of cluster heads in DEEC (Distributed Energy Efficient Clustering algorithm) [23]. There are two types of nodes used in the network named as advanced node and normal nodes. The advance nodes are having more energy than the normal nodes by $\alpha$ time and $m$ is the fraction of advanced nodes present in the entire WSN. The value $p_{\text {opt }}$ is the reference value of the average probability $p_{i}$. Let $\mathrm{E}_{\mathrm{i}}(\mathrm{r})$ denotes the energy of a node $S_{i}$ at round $\mathrm{r}$. A weight is 
added with the probability value for the cluster head for these heterogeneous nodes. The weighted probabilities for advanced nodes $\left(P_{a d v}\right)$ and normal nodes $\left(P_{n r m}\right)$ are as follows.

$$
\begin{aligned}
& P_{a d v}=\frac{P_{o p t}}{1+\alpha m} \\
& P_{n r m}=\frac{P_{o p t}(1+\alpha)}{1+\alpha m}
\end{aligned}
$$

The value of probability ratio $\left(p_{i}\right)$ to be a cluster head for the current round is

$$
p_{(i)}= \begin{cases}\frac{p_{o p t} E_{i}(r)}{(1+a m)_{E}^{-}(r)} & \text { if } \mathrm{s}_{i} \text { is the normal node } \\ \frac{p_{o p t}(1+a) E_{i}(r)}{(1+a m)_{E}^{-}(r)} & \text { if } \mathrm{s}_{i} \text { is the advanced node }\end{cases}
$$

where $\overline{\mathrm{E}}(\mathrm{r})$ is the average energy of the network at round $\mathrm{r}$. This $p_{i}$ can be used in calculating the probability threshold as

$$
T_{\left(s_{i}\right)}= \begin{cases}\frac{P_{i}}{1-P_{i}\left(\bmod \frac{1}{P_{i}}\right)} & \text { if } s_{i} \in G \\ 0 & \text { Otherwise }\end{cases}
$$

where $\mathrm{G}$ is the number of nodes that are eligible to become cluster head at round $\mathrm{r}$. In DEEC, the energy consumption of each node is consistent and the distribution of cluster head is also made uniformly. The cluster heads elected using these protocols are sometimes closely placed to each other. This will affect the equilibrium of the network therefore the network life time is restricted. This problem has been solved in [22]. Takehiro Furuta el al. proposed an algorithm using facility location theory [25] to select optimum number of cluster heads. Since the reactive wireless sensor networks are considered in this work, the number of active nodes and alive nodes are play a significant role in the computation of optimum number of cluster.

\section{Proposed methodology}

\subsection{Network model}

The homogeneous WSNs are considered for the simulation. There is no physical or hardware difference between cluster head and cluster member in this simulation environment. All the nodes considered in the WSN are having equal initial energy and the nodes are assumed that they are having similar functionality. We The first order radio model is considered for the simulation [24]. The first order radio model is classified into free space model and multi path fading model based on the distance between the source and destination. Let $d_{o}$ be the threshold value for deciding the type of model to be used in the communication. The energy used for sending the data among the two models is depending on the value $d_{o}$. The $d_{o}$ can be calculated as

$$
d_{o}=\sqrt{\frac{\varepsilon_{f s}}{\varepsilon_{m p}}}
$$

where $\varepsilon_{\mathrm{fs}}$ and $\varepsilon_{\mathrm{mp}}$ are the energy usage associated with free space and multipath fading model respectively.

Transmitting energy: 
Let $\mathrm{E}_{\mathrm{TX}}$ be the energy required for sending a packet of size $\mathrm{K}$ over a distance $d$. The energy $\mathrm{E}_{\mathrm{TX}}$ is calculated based on the distance $d$ and $d_{o}$. If $d \leq d_{o}$, the free space model is used and for the other case the multi path fading model is applied.

$$
\begin{aligned}
& \text { If } d \leq d_{o} \text { then } \mathrm{E}_{\mathrm{TX}}=\left(\mathrm{E}_{\text {elec }} * \mathrm{~K}\right)+\left(\varepsilon_{\mathrm{fs}} * \mathrm{~K} * d^{2}\right) \\
& \text { If } d>d_{o} \text { then } \mathrm{E}_{\mathrm{TX}}=\left(\mathrm{E}_{\text {elec }} * \mathrm{~K}\right)+\left(\varepsilon_{\mathrm{mp}} * \mathrm{~K} * d^{4}\right)
\end{aligned}
$$

where $E_{\text {elec }}=E_{t x}+E_{A G}$. In the case of cluster head, $E_{A G}$ is the energy spent for data aggregation and for the normal sensor nodes $\mathrm{E}_{\mathrm{AG}}$ will be zero. The value $\mathrm{E}_{\text {elec }}$ is the energy used for sending a bit $/ \mathrm{m}^{2}$.

\section{Receiving energy:}

Let $\mathrm{E}_{\mathrm{RX}}$ be the energy needed for receiving a packet of size $\mathrm{K}$ for a distance $d$.

$$
\mathrm{E}_{\mathrm{RX}}=\left(\mathrm{E}_{\mathrm{rx}} * \mathrm{~K}\right)
$$

where $E_{\mathrm{rx}}$ is the energy used for receiving a bit $/ \mathrm{m}^{2}$.

\subsection{EGEC Methodology}

In the Reactive Wireless Sensor Networks, the nodes get activated when the sensed value exceeds the threshold value. In applications using reactive wireless sensor networks with large number of nodes, all the nodes are not activated simultaneously. Applications like fire detection and temperature monitoring, the sensor nodes in and around the fire affected and high temperature area are activated respectively. These nodes are triggered to send data due to surpassing of the sensed parameter beyond the threshold values. At that time, the remaining sensor nodes are under idle state. Therefore it is observed that the probability ratio $p_{o}$ for deciding the number of clusters used in earlier protocols is not feasible to give better cluster count. Therefore in this work, the number of alive nodes and number of active nodes at the corresponding round are considered for finding the number of clusters.. Let $m$ be the total number of active nodes at a particular round and $n$ represents the total number of alive nodes in the network, then

$$
\begin{aligned}
& x=\left[\frac{m}{n} * 10\right] \\
& y=1-e^{-0.6 x}
\end{aligned}
$$

Let $\mathrm{CH}_{\text {cnt }}$ represents the new cluster head count, then

$$
C H_{c n t}=\left[p_{o} * n * y\right]
$$

Using the equation (10), the weight is calculated as

$$
p_{\text {wgt }}=\left\{\begin{array}{lr}
p_{o}-\mathrm{CH}_{\text {cnt }}, & \text { if } \mathrm{CH}_{\text {cnt }}<p_{o} \\
\mathrm{CH}_{\text {cnt }}-p_{o \prime} & \text { Otherwise }
\end{array}\right.
$$

where $p_{o}$ is the probability ratio decided initially. The probability ratio $p_{i}$ for the current round of operation $i$ is calculated as follows.

$$
p_{i}=\left\{\begin{array}{lr}
p_{o}-p_{w g t \prime} & \text { if } C H_{c n t}<p_{o} \\
p_{o}+p_{w g t \prime} & \text { Otherwise }
\end{array}\right.
$$


The probability ratio for the current round is replaced by the $p_{i}$ value. The remaining operations are executed as in TEEN protocol.

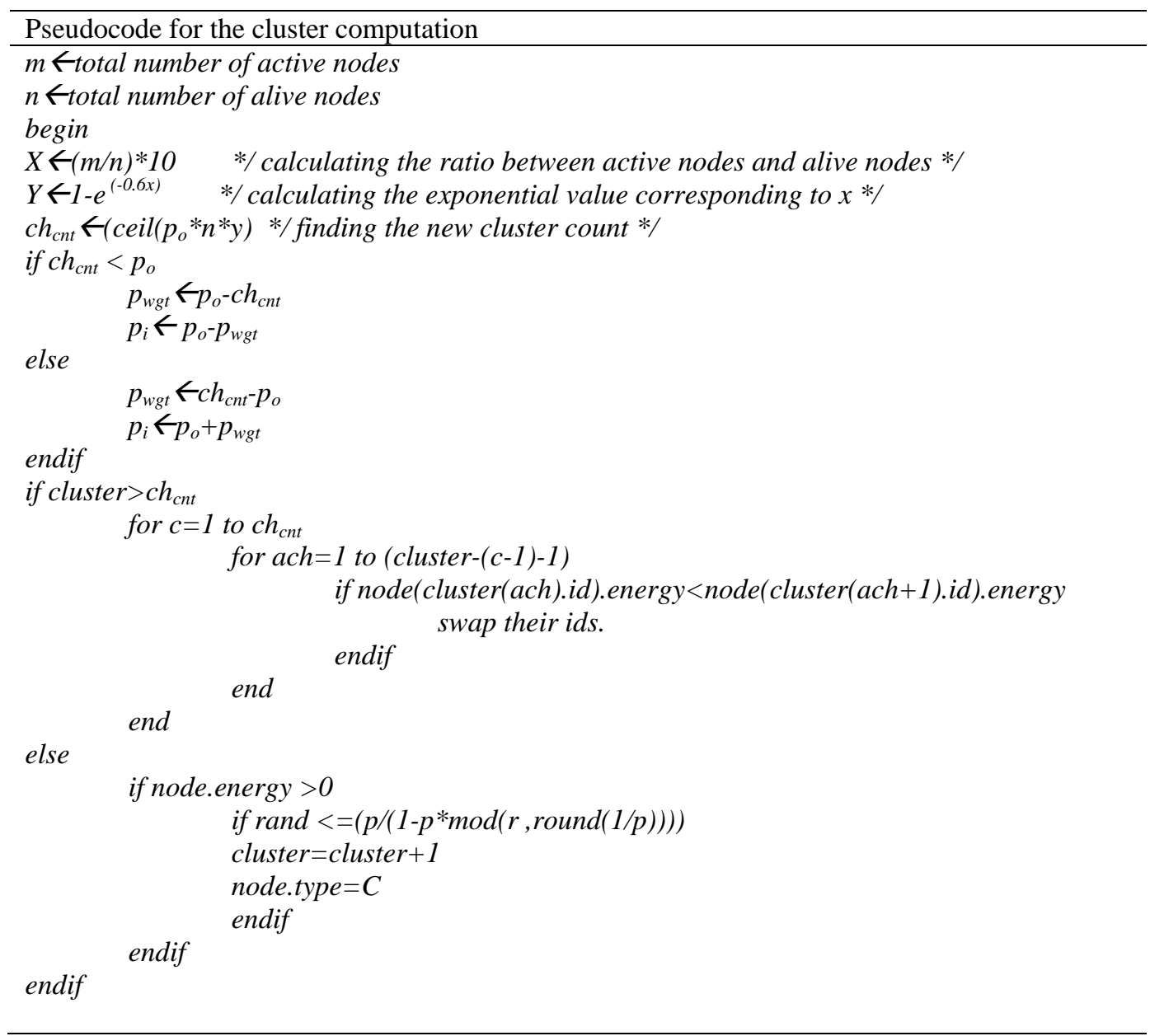

\section{Simulation Results}

The TEEN extension has been used for the simulation and the simulation was performed in MATLAB R2013a. Modification was made on the available TEEN extension. Simulations were performed with different parameter values and at various scenarios. Experimental results show that the network life time and throughput are increased when compared with the existing TEEN protocol. A simulation environment with field dimension of $100 \mathrm{~m} \times 100 \mathrm{~m}$ has been considered for the experiment. The total number of sensor nodes deployed in the network is 1000. The network parameters of the sensor network are given in Table 1.

\subsection{Performance metrics}

The following metrics are selected to measure the performance of the EGEC methodology with the existing TEEN protocol.

a) Network Life time 
It is the time duration between the starting time of the sensing and sending data by sensor nodes (beginning of the network) and the death of the last live node.

\section{b) Throughput}

It is the total amount of data received by the base station from the sensor nodes in a WSN.

\section{c) Stability period}

Stability period is the time or number of rounds between the start of the network and death of the first sensor node.

Table 1. Network Parameters used in Simulation

\begin{tabular}{|c|c|}
\hline Parameters & Values \\
\hline Sink Position & $50 * 50$ \\
\hline efs(Amplifier used in free space model) & $10 * 10^{-12} \mathrm{j}$ \\
\hline$\varepsilon m p$ (Amplifier used in multipath fading model) & $0.0013 * 10^{-12} \mathrm{j}$ \\
\hline $\mathrm{E}_{\mathrm{AG}}$ (Aggregation Energy) & $5 * 10^{-9} \mathrm{j}$ \\
\hline Initial Energy $E_{o}$ & $0.01 \mathrm{j}$ \\
\hline Packet Length & 2000bits \\
\hline Control Packet Length & 100bits \\
\hline Probability of $\mathrm{CHs}\left(\mathrm{P}_{\mathrm{o}}\right)$ & 0.1 \\
\hline Maximum rounds & 150 \\
\hline No of Nodes(n) & 1000 \\
\hline
\end{tabular}

Number of iterations are performed to find the optimum value in the expression $1-\mathrm{e}^{-0.6 \mathrm{x}}$ in such a way that it gives a better network life time and throughput. Initially the equation is considered as $1-\mathrm{e}^{-\$ \mathrm{x}}$ and value ranging from 0.1 to 0.9 is assigned for $\$$ during different iterations to find the optimum value which gives the better network life time. It is found that 0.6 is the optimum value by varying the value from 0.1 to 0.9 . Figure 1a) show the performance of the protocol with $\$$ has the value 0.3, 0.6 and 0.9. From the graph it is understood that the expression 1-e $\mathrm{e}^{-0.6 \mathrm{x}}$ has given the better result than the other values. Figure 1b) show the throughput analysis of the network. It indicates that the number of data packets send to the base station is more after 80 rounds while using EGEC protocol than that of TEEN. Since the optimum number of clusters is formed, more number of nodes become cluster head is avoided. Therefore these nodes lifetime is extended which in turn improve the overall network lifetime.

Figure 2 show the lifetime of the network during simulation using EGEC and TEEN. EGEC uses optimum number of clusters so that excess cluster formation is avoided. The number of clusters is optimized so that the unselected cluster heads are assigned as normal nodes. The energy of these nodes is conserved so that the network life time is increased. The total number of nodes alive in the network is more when using the EGEC protocol than using the TEEN protocol. The graph indicates that the number of alive nodes is more even after 80 rounds of iterations are performed. Thus the network works in a better way even in critical situations. The number of clusters will be calculated in each round of operation based on the probability ratio $p_{i}$. 


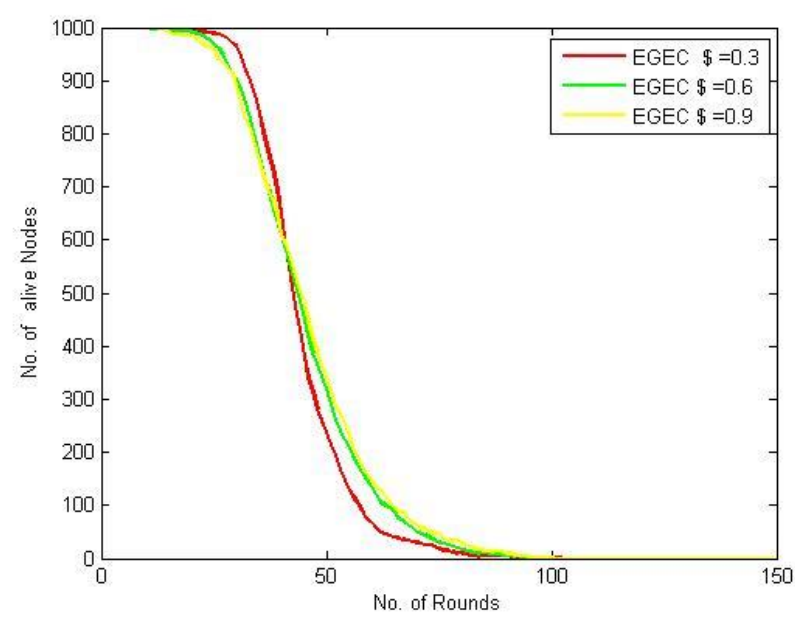

(a)

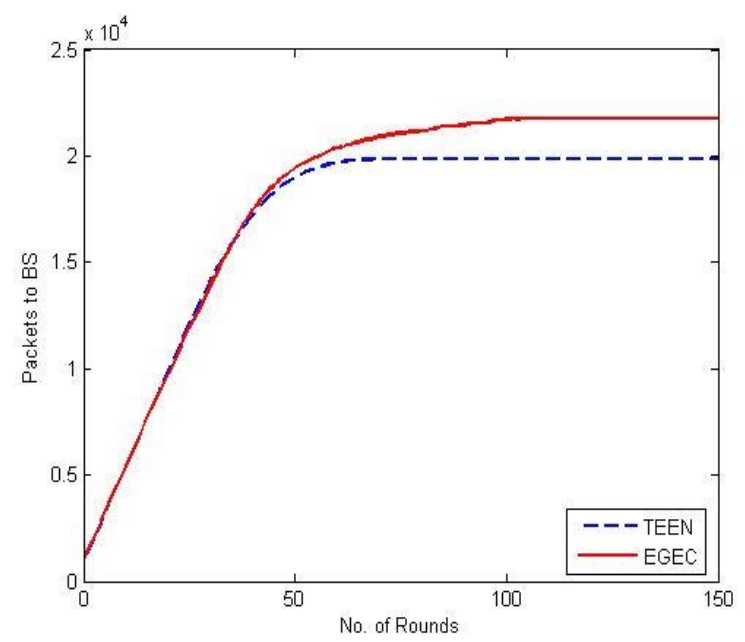

(b)

Fig.1. (a) Network Lifetime when $\$=0.3,0.6$ and 0.9 , (b) Throughput analysis between TEEN and EGEC

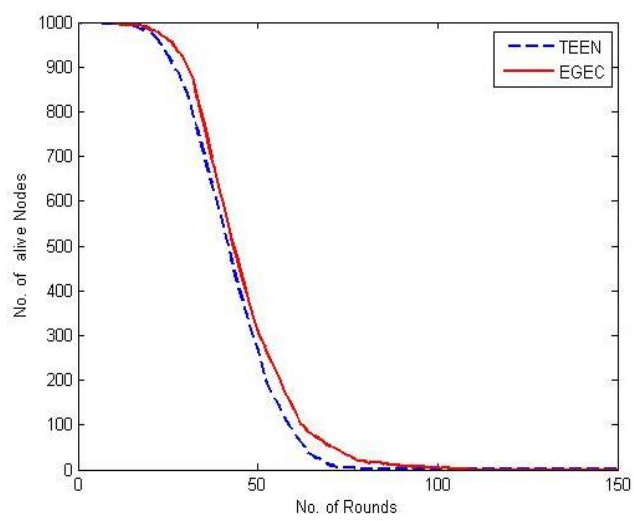

Fig.2. Network Life Time analysis between TEEN and EGEC

Figure 3a) analyzed the performance of TEEN and EGEC in terms of Network lifetime with different number of sensor nodes deployed in the network. The number of sensor nodes installed in the network has been increased and the life time of the network is analyzed. From the analysis, it is observed that EGEC performed better for different number of nodes in the network. Figure 3b) show the throughput of the network for the different number of sensor nodes in the network. Since the number of clusters assigned is based on the active nodes and alive nodes, the optimum cluster heads are generated. Hence the life time of the network is increased which in turn improves the throughput. From this analysis it is observed that EGEC has produced better throughput and elongates the life time of the network than TEEN. 


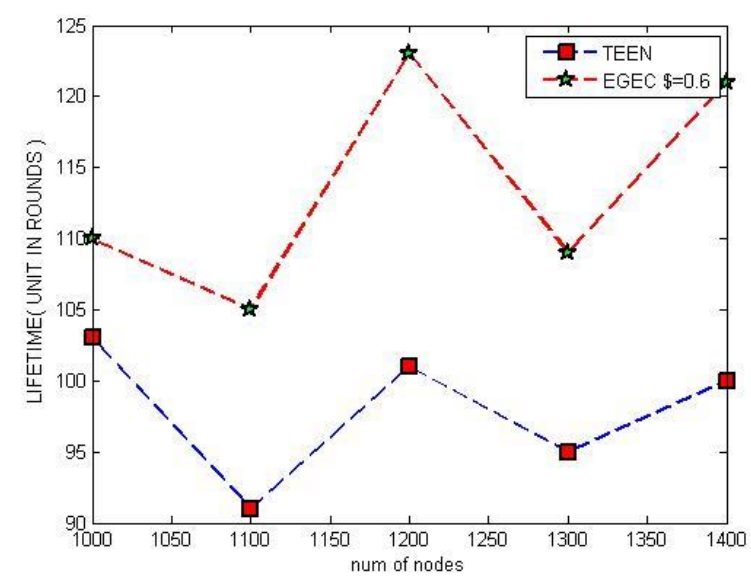

(a)

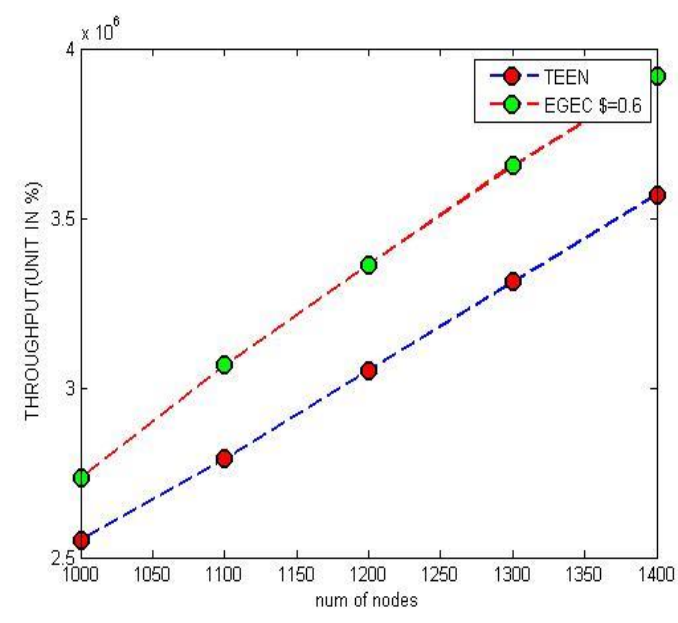

(b)

Fig.3. (a) Network Lifetime for WSN with various nodes, (b) Throughput for WSN with various nodes

\section{Conclusions}

In this paper, a new methodology called EGEC is proposed to conserve energy of the reactive sensor networks. The number of clusters for the reactive sensor networks is optimally calculated in EGEC based on active nodes and alive nodes at the corresponding round with the exponential function. Considerable amount of energy in the nodes of the WSN is preserved by optimally computing the clusters. The simulation results show that the performance of the proposed protocol is improved in terms of throughput and network lifetime. In this work, only the number of active nodes and alive nodes are considered for cluster count computation. The change of energy from previous round to current round and the weight value of the nodes are also taken into consideration for the cluster head estimation.

\section{References}

[1] Khedr, Ahmed M., and Walid Osamy. "Effective target tracking mechanism in a self-organizing wireless sensor network." Journal of Parallel and Distributed Computing 71.10 (2011): 1318-1326.

[2] Cerpa, Alberto, et al. "Habitat monitoring: Application driver for wireless communications technology." ACM SIGCOMM Computer Communication Review 31.2 supplement (2001): 20-41.

[3] Estrin, Deborah, et al. "Next century challenges: Scalable coordination in sensor networks." Proceedings of the 5th annual ACM/IEEE international conference on Mobile computing and networking. ACM, 1999.

[4] Akyildiz, Ian F., et al. "A survey on sensor networks." Communications magazine, IEEE 40.8 (2002): 102-114.

[5] Sheng, Zhengguo, et al. "A survey on the ietf protocol suite for the internet of things: Standards, challenges, and opportunities." Wireless Communications, IEEE 20.6 (2013): 91-98.

[6] Acampora, Giovanni, et al. "A survey on ambient intelligence in healthcare." Proceedings of the IEEE 101.12 (2013): 2470-2494.

[7] Zeng, Yuanyuan, et al. "Directional routing and scheduling for green vehicular delay tolerant networks." Wireless networks 19.2 (2013): 161-173.

[8] Chen, Min, et al. "Body area networks: A survey." Mobile networks and applications 16.2 (2011): 171193. 
[9] Nakamura, Eduardo F., Antonio AF Loureiro, and Alejandro C. Frery. "Information fusion for wireless sensor networks: Methods, models, and classifications." ACM Computing Surveys (CSUR) 39.3 (2007): 9.

[10] Wei, Guiyi, et al. "Prediction-based data aggregation in wireless sensor networks: Combining grey model and Kalman Filter." Computer Communications 34.6 (2011): 793-802.

[11] Xiang, Liu, Jun Luo, and Athanasios Vasilakos. "Compressed data aggregation for energy efficient wireless sensor networks." Sensor, mesh and ad hoc communications and networks (SECON), 2011 8th annual IEEE communications society conference on. IEEE, 2011.

[12] Anastasi, Giuseppe, et al. "Energy conservation in wireless sensor networks: A survey." Ad hoc networks 7.3 (2009): 537-568.

[13] Liu, Xuxun. "A survey on clustering routing protocols in wireless sensor networks." Sensors 12.8 (2012): 11113-11153.

[14] Younis, Ossama, and Sonia Fahmy. "HEED: a hybrid, energy-efficient, distributed clustering approach for ad hoc sensor networks." Mobile Computing, IEEE Transactions on 3.4 (2004): 366-379.

[15] Chamam, Ali, and Samuel Pierre. "A distributed energy-efficient clustering protocol for wireless sensor networks." Computers \& electrical engineering 36.2 (2010): 303-312.

[16] Smaragdakis, Georgios, Ibrahim Matta, and Azer Bestavros. "SEP: A stable election protocol for clustered heterogeneous wireless sensor networks." Second international workshop on sensor and actor network protocols and applications (SANPA 2004). Vol. 3. 2004.

[17] Rajagopalan, Ramesh, and Pramod Varshney. "Data-aggregation techniques in sensor networks: a survey." IEEE Communications Surveys \& Tutorials 4.8 (2006): 48-63.

[18] Heinzelman, Wendi B., Anantha P. Chandrakasan, and Hari Balakrishnan. "An application-specific protocol architecture for wireless microsensor networks." Wireless Communications, IEEE Transactions on 1.4 (2002): 660-670.

[19] Lindsey, Stephanie, and Cauligi S. Raghavendra. "PEGASIS: Power-efficient gathering in sensor information systems." Aerospace conference proceedings, 2002. IEEE. Vol. 3. IEEE, 2002.

[20] Manjeshwar, A., and D. P. Agrawal. "TEEN: a routing protocol for enhanced efficiency in wireless sensor networks." Parallel and Distributed Processing Symposium., Proceedings 15th International. 2001.

[21] Manjeshwar, Arati, and Dharma P. Agrawal. "APTEEN: A hybrid protocol for efficient routing and comprehensive information retrieval in wireless sensor networks." ipdps. IEEE, 2002.

[22] Sundareswaran, P., K. N. Vardharajulu, and R. S. Rajesh. "DECH: Equally Distributed Cluster Heads Technique for Clustering Protocols in WSNs." Wireless Personal Communications 84.1 (2015): 137-151.

[23] Qing, Li, Qingxin Zhu, and Mingwen Wang. "Design of a distributed energy-efficient clustering algorithm for heterogeneous wireless sensor networks." Computer communications 29.12 (2006): 22302237.

[24] Liu, Yuhua, Yongfeng Zhao, and Jingju Gao. "A new clustering mechanism based on LEACH protocol." Artificial Intelligence, 2009. JCAI'09. International Joint Conference on. IEEE, 2009.

[25] Furuta, Takehiro, et al. "A new clustering model of wireless sensor networks using facility location theory." Journal of the Operations Research Society of Japan 52.4 (2009): 366-376.

\section{Authors' Profiles}

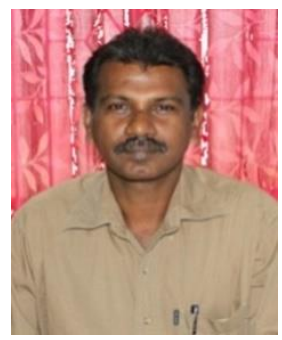

P. Sundareswaran is currently working as Assistant Professor in the Department of Computer Science \& Engineering in Manonmaniam Sundaranar University, Tirunelveli, India. He is pursuing his Ph.D in the area of Wireless Sensor Networks. He received his M.E. degree in Computer Science and Engineering from Manonmaniam Sundaranar University Tirunelveli, India in 2004. His areas of interest in research are Computer Networks, Mobile Computing, IoT and Wireless communications 


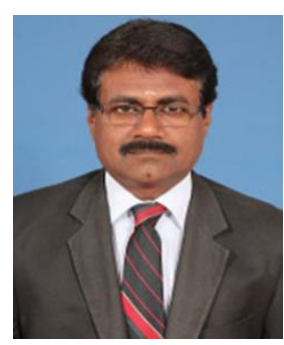

Dr. R. S. Rajesh received his B. E and M. E degrees in Electronics and Communication Engineering from Madurai Kamaraj University, Madurai, India in the year 1988 and 1989 respectively. He got his Ph.D degree from Manonmaniam Sundaranar University during 2004. He is currently the Professor of Department of Computer Science and Engineering, Manonmaniam Sundaranar University. He has 22 years of PG teaching experience. He has published 100 articles in leading international journals. His research areas include Vehicular Adhoc Networks, Wireless networks, Digital image processing and Pervasive computing

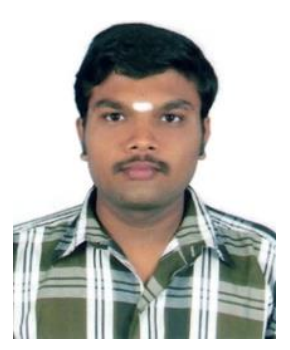

K. N. Vardharajulu was a P. G. student in Department of Computer Science and Engineering in Manonmaniam Sundaranar University. He received his B.Tech degree in Software Engineering from Anna University Tirunelveli, India in 2008. His research interests include wireless sensor/adhoc networks, Digital image processing and data mining. He is currently focusing on routing protocols for wireless sensor networks

How to cite this paper: Sundareswaran P, Rajesh R S, Vardharajulu K N, "EGEC: An Energy Efficient Exponentially Generated Clustering Mechanism for Reactive Wireless Sensor Networks", International Journal of Wireless and Microwave Technologies(IJWMT), Vol.8, No.6, pp. 62-72, 2018.DOI: 10.5815/ijwmt.2018.06.05 